\title{
Anaphylaxis to Skin Prick Testing in a 29-Year-Old Woman
}

\author{
Caroline J. Lodge ${ }^{1}$, Shyamali C. Dharmage ${ }^{1,2}$, Rida U. Khalafzai ${ }^{1}$, Michael J. Abramson ${ }^{3}$, \\ David J. Hill' ${ }^{2}$, Cliff S. Hosking ${ }^{4}$, Katrina J. Allen ${ }^{2,5,6}$
}

${ }^{1}$ Centre for Molecular, Environmental, Genetic and Analytic Epidemiology, School of Population Health, University of Melbourne, Melbourne, Australia; ${ }^{2}$ Murdoch Children's Research Institute, Royal Children's Hospital, Melbourne, Australia; ${ }^{3}$ Department of Epidemiology and Preventive Medicine, Monash University, The Alfred Hospital, Melbourne, Australia; ${ }^{4}$ John Hunter Children's Hospital, Newcastle, Australia; ${ }^{5}$ Department of Allergy, Royal Children's Hospital, Parkville, Australia; ${ }^{6}$ Department of Paediatrics, Royal Children's Hospital, Parkville, Australia.

Email: clodge@unimelb.edu.au

Received August $10^{\text {th }}, 2012$; revised September $24^{\text {th }}, 2012$; accepted October $7^{\text {th }}, 2012$

\begin{abstract}
Introduction: Skin prick testing (SPT) is a common test to diagnose IgE sensitization and the rate of adverse reactions is very low. Case Presentation: 29-year-old woman with anaphylactic reaction to routine SPT with 11 allergens (aero and food). Discussion: Allergy profile included multiple food and inhalant allergies, polysensitization, prior episode of anaphylaxis to mushrooms, active eczema and active hay fever but not active asthma. Conclusion: Anaphylaxis to SPT, although a rare event, is unpredictable necessitating availability of appropriate personnel and adequate resuscitation facilities for all testing.
\end{abstract}

Keywords: Anaphylaxis; Skin Prick Testing

\section{Introduction}

Skin prick testing (SPT) is commonly used to diagnose IgE mediated sensitizations to environmental allergens and used extensively in allergy research. Its safety profile has been the subject of several reviews [1-4], reporting a low incidence of adverse events when using standardized commercially available diagnostic extracts.

Since 1980 there have been 17 case reports of anaphylaxis and 7 recorded fatalities from skin testing, however 6 of these fatalities involved intradermal testing and one scratch testing [5]. Over this time, there has never been a fatality reported from prick testing. The incidence of any adverse reaction is low, $0.24 \%$ from the largest prospective study [3]. Of these, only half were generalized allergic reactions, the remainder attributed to vasovagal causes.

\section{Case Report}

A female pharmacy assistant, aged 29, underwent SPT in our research clinic, using single tine Lancets, to 11 allergens (Cow's milk, egg white, peanut, cashew, shrimp, rye grass, cat pelt, mixed grasses, Alternaria tenuis, Penicillium mix and house dust mite [ALK-Abello, Hørsholm DK; Hollister-Stier, Spokane WA]). She had a history of multiple allergies including: anaphylaxis following mushroom ingestion aged 23 (manifest by diffuse rash, coughing, throat swelling); sensitization to dust mite, cat and rye grass for which she underwent 1 year of sublingual immunotherapy (SLIT) 10 years previously; and reported allergy to rice aged 16 years (manifest by immediate rash and vomiting). She also reported previous SPT sensitization to unspecified nuts.

Additionally, she described: allergic rhinitis from age 14 , on exposure to cats, feathers, dust or pollens and had current symptoms; generalized eczema from age 16 with 3 episodes in the past year, and; a history of wheeze aged 8 - 16 years, with no wheezy episodes in the past year.

Her medications included daily use of Indomethacin and paracetamol for arthritis and as needed use of medications for hay fever (fluticasone nasal, cetirizine, loratidine, fexofenadine or promethazine), eczema (mometasone) and cough (dextromethorphan). According to her survey responses, her arthritis has not been diagnosed as being of autoimmune aetiology.

Five minutes after SPT the participant had mild coughing. After 10 minutes she complained of itchy throat, watery and itchy eyes and cough became continuous. She then mentioned chest tightness and swollen tongue. Persistent cough, difficulty breathing and swelling of the tongue are all consistent with the definition of anaphylaxis issued by the Australasian society of clinical immunology and allergy. Immediately following the onset 
of sustained coughing she was administered oxygen via facemask and $10 \mathrm{mg}$ of oral cetirizine hydrochloride and shortly after this nebulized adrenaline was given by the emergency response team. Her symptoms resolved over the ensuing 20 minutes. After observation for 4 hours she was discharged home without further event.

The results of tests performed earlier in the research clinic showed a mildly increased expired nitric oxide level of 34 (normal 20 - 30 parts per billion) and a normal pre and post bronchodilator (salbutamol) spirometry. Wheal sizes for allergens other than histamine $(3.5 \mathrm{~mm})$ were not recorded.

\section{Discussion}

We report an anaphylactic reaction following SPT to food and aero-allergens in a 29-year-old woman with one previous episode of food related anaphylaxis (presumed mushroom allergy), multiple prior inhalant and food allergies and active rhinitis and eczema. The episode resolved quickly with appropriate treatment. Unfortunately, the participant refused any further investigations of this episode so we were unable to further elucidate the likely cause.

Recent articles [2-4,6] have identified young age, active eczema, current asthma, high degree of reactivity, polysensitization, testing with fresh food extracts, previous systemic reaction to SPT, duplicate testing and pregnancy as high risk features for anaphylaxis from skin prick testing. Our subject had two of these (active eczema and previous polysensitization) but was similar to the other 1375 participants of a high allergy risk cohort who had been tested over the length of this study without anaphylactic responses. Of particular note, she had no active asthma and spirometry testing immediately prior to the anaphylaxis was normal.

She had experienced an episode of food related anaphylaxis aged 23, presumed due to mushrooms. Mushroom is a rare, severe form of allergy [7], more common in workers on mushroom farms exposed to high levels of spores. There is some evidence of cross over reactivity between moulds (including Alternaria) and mushroom species [8] and this is the most likely cause of anaphylaxis in our participant, however other factors in her history may be relevant.

Although nut sensitivity was reported in the past, there had never been any documented reaction on previous nut exposure, so we consider this a less likely cause.

She had previously undergone SLIT to aeroallergens. Adverse reactions have been described for food allergens after discontinuation of therapy and re-exposure to the allergen. The safety profile of SLIT is impressive [9] and no reports have been made of this nature, however, her length of treatment would now be considered sub-optimal (1 year instead of the 3 years currently recommended) and the period since cessation is longer than the follow-up reports from any of the RCTs.

She also reported using a cough suppressant containing dextromethorphan. Recent reports have implicated pholcodine in cough suppressants as responsible for inducing IgE mediated anaphylaxis to neuromuscular blocking agents used in anaesthesia, and a reduction in this phenomenon has been seen since withdrawal of this product from the Norwegian market [10]. Pholcodine use has also been shown to cause a rise in total IgE. Other morphine derivatives such as dextromethorphan may have similar effects on IgE production and facilitate a hypersensitivity response to tested allergens.

Laryngospasm can be difficult to distinguish from anaphylaxis; however, the more gradual onset and length of this episode, along with the prominence of cough and absence of stridor make laryngospasm a less likely cause.

Despite large review series and sporadic case reports in the literature, there is still no good method to identify those at high risk of anaphylaxis from SPT using commercial extracts. In our case report, the most prominent allergic symptom prior to SPT was rhinitis which has not previously been described as a high risk feature for anaphylaxis from skin prick testing

\section{Conclusion}

Prior to skin prick testing there were no obvious indicators to identify this participant as being at higher risk than others in our high-risk cohort. The anaphylactic episode was unpredictable. Although the occurrence of anaphylaxis in the context of SPT is rare and the resolution in all reported cases has been quick and non-fatal, the risk of anaphylaxis still exists and the current inability to predict who may be affected means that resuscitation facilities should be available for all SPT.

\section{REFERENCES}

[1] P. C. Turkeltaub and P. J. Gergen, "The Risk of Adverse Reactions from Percutaneous Prick-Puncture Allergen Skin Testing, Venipuncture, and Body Measurements: Data from the Second National Health and Nutrition Examination Survey 1976-1980 (NHANES II)," Journal of Allergy and Clinical Immunology, Vol. 84, No. 6, 1989, pp. 886-890. doi:10.1016/0091-6749(89)90384-9

[2] E. Novembre, R. Bernardini, G. Bertini, G. Massai and A. Vierucci, "Skin Prick Test Induced Anaphylaxis," Allergy, Vol. 51, 1995, pp. 60-61.

[3] G. Norrman and K. Fälth-Magnusson, "Adverse Reactions to Skin Prick Testing in Children-Prevalence and Possible Risk Factors," Pediatric Allergy and Immunology, Vol. 20, No. 3, 2009, pp. 273-278. doi:10.1111/j.1399-3038.2008.00761.x

[4] G. Liccardi, A. Salzillo and G. D'Amato, "The Risk of Generalized Allergic Reactions to Skin Prick Testing May Be Higher in Poly-Sensitized Individuals and in 
Those Who Underwent SPT with Fresh Foods," Pediatric Allergy and Immunology, Vol. 21, 2010, pp. 656-657. doi:10.1111/j.1399-3038.2009.00984.x

[5] G. Liccardi, G. D’Amato, G. Walter Canonica, A. Salzillo, A. Piccolo and G. Passalacqua, "Systemic Reactions from Skin Testing: Literature Review," Journal of Investigantional Allergology and Clinical Immunology, Vol. 16, 2006, pp. 75-78.

[6] M. A. Valyasevi, D. E. Maddox and J. T. C. Li, "Systemic Reactions to Allergy Skin Tests," Annals of Allergy, Asthma \& Immunology, Vol. 83, No. 2, 1999, pp. 132136.

[7] R. Torricelli, S. G. O. Johansson and B. Wüthrich, "Ingestive and Inhalative Allergy to the Mushroom Boletus Edulis," Allergy, Vol. 52, No. 7, 1997, pp. 747-751. doi:10.1111/j.1398-9995.1997.tb01232.x

[8] I. Herrera-Mozo, B. Ferrer, J. L. Rodriguez-Sanchez and C. Juarez, "Description of a Novel Panallergen of CrossReactivity between Moulds and Foods," Immunological Investigations, Vol. 35, No. 2, 2006, pp. 181-197. doi:10.1080/08820130600616599

[9] S. Y. Lin and B. Leatherman, "Sublingual Immunotherapy," Otolaryngologic Clinics of North America, Vol. 44, No. 3, 2011, pp. 753-764. doi:10.1016/j.otc.2011.03.012

[10] E. Florvaag, S. G. O. Johansson, Å. Irgens, G. H. de Pater, "IgE-Sensitization to the Cough Suppressant Pholcodine and the Effects of Its Withdrawal from the Norwegian Market," Allergy, Vol. 66, No. 7, 2011, pp. 955-960. doi:10.1111/j.1398-9995.2010.02518.x 